\title{
Distortion Operator of Uncertainty Claim Pricing Using Weibull Distortion Operator
}

\section{Akintunde OA* $^{*}$}

Department of Mathematics, Faculty of Science, Federal University Oye Ekiti, Ekiti State, Nigeria

\begin{abstract}
The problem of uncertainty claim pricing using distortion operators is considered in this research paper. This approach was first developed in insurance pricing, where the original distortion function was defined in terms of the normal probability distribution. This approach is generalized by using a distortion that is based on the Weibull distribution in this research paper. The Weibull family allows for heavier and skewed tail because it is so flexible that other statistical distributions can be recovered from it by change of parameters. The problem of uncertainty claims has been extensively studied for non-Gaussian model in which the formula was derived for the normal Inverse Gaussian distribution Asset pricing. It is shown in this paper how Weibull based distortion function can used to derive the formula for asset pricing of uncertainty future returns of a risky asset. The risk measure for the incurred risk modelled by the Weibull variables was derived and it was shown that it follows the power law.
\end{abstract}

Keywords: Uncertainty claims; Weibull distribution; Insurance; Pricing; Power law

\section{Introduction}

Distortion risk measures are used to price financial and insurance risk and estimate the returns of most financial assets [1]. This is done by adjusting the true probability to give more weight to higher risks events.

They are used to determine the future price in respect of a random loss variable $x$ with the view to avoid insolvency [2]. The future returns for the risky assets is the expected value under the distorted probability which is called the risk adjusted measure. Distortion risk measure predicts the price of return for a given risk portfolio, based on its downside risk potentials [3].

A Distortion risk measure can be defined as the distorted expectation of any non-negative loss random variable $X$ [4]. It is accomplished by using a "dual utility" or the distortion function $g$ as follows:

$$
\operatorname{Pr}\left(g(x)=\int_{0}^{\infty} g\left(1-f_{x}(x)\right) d x=\int_{0}^{\infty} g\left(s_{x}(x)\right) d x\right.
$$

Where $\mathrm{g}:[0,1] \rightarrow[0,1]$ is a continuous increasing function with $g(0)=0$ and $g(1)=1[5]$.

$F_{x}(x)$ denotes the cumulative distribution function of $x$ and $g\left(F_{x}(x)\right)$ is the distorted distribution function [6].

The survival function is given as;

$$
S_{x}(x)=1-f_{x}(x)=\operatorname{Pr}(\mathrm{X}>x)
$$

\section{Properties of Distortion Risk Measures}

The Properties of distortion risk measures as stated by Artzner et al. are given below:

1. Monotocity: if $x \geq 0$, then $\operatorname{Pr}(g(x)) \geq 0$

2. Positive homogeneity: $[g(\lambda x)=\lambda \operatorname{Pr}[g(x)], \forall \lambda \geq 0$

3. Translate invariance: $[g(x+c)]=\operatorname{Pr}[g(x)]+c, \forall c \in \mathbb{R}$

4. $\operatorname{Pr}[g(-x)]=-\operatorname{Pr}[g(-x)]$ where $g(x)=1-g(1-x)$

5. If a random variable $x_{n}$ has a finite number of values, that is a. $\left(x_{n} \rightarrow x\right)$, and if $\operatorname{Pr}(g(x))$ exits, then $\operatorname{Pr}(g(x)) \rightarrow \operatorname{Pr}(g(x))$

6. Comonotic additivity: If $\mathrm{x}$ and $\mathrm{y}$ are comonotic risk taking positive and negative values, then: $\operatorname{Pr}[g(x+y)]=\operatorname{Pr}[g(x)]+\operatorname{Pr}[g(y)]$

7. In general cases, distortion risk measures are not additive

a. $\operatorname{Pr}[g(x+y)] \neq \operatorname{Pr}[g(x)]+\operatorname{Pr}[g(y)]$

8. Sub-additivity: If the distortion function $g(x)$ is concave, then

a. $\operatorname{Pr}[g(x+y)] \leq \operatorname{Pr}[g(x)]+\operatorname{Pr}[g(y)]$.

9. For a non-decreasing distortion function $\mathrm{g}$, the associated risk measure $\operatorname{Pr}(g)$ is consistent with the stochastic dominance of order one. That is, if $x \leq y$, then $\operatorname{Pr}[\mathrm{g}(x)] \leq \operatorname{Pr}[\mathrm{g}(y)]$

10. For non-decreasing concave distortion function $g$, the associated risk measure $\operatorname{Pr}(g)$ is consistent with the stochastic dominance of order two. That is, if $x<y$ then: $\operatorname{Pr}[\mathrm{g}(x)] \leq \operatorname{Pr}[\mathrm{g}(y)]$.

11. For a strictly concave function $\mathrm{g}$, the associated risk measure $\operatorname{Pr}(g)$ is consistent with the stochastic dominance of order 2 , if for $x>y$ then $\operatorname{Pr}[\mathrm{g}(x)]<\operatorname{Pr}[\mathrm{g}(y)]$.

\section{Example of distortion risk measure}

The mathematical expectation of $g(x)=x$ if it exists is given as:

$$
\mathrm{E}(x)=\operatorname{Pr}[g(x)]
$$

And the variance at risk $(\mathrm{VaR})$ corresponds to the distortion given as follows:

*Corresponding author: Akintunde OA, Department of Mathematics, Faculty of Science, Federal University Oye Ekiti, Ekiti State, Nigeria, Tel: +2348034253309, E-mail: oyetunde.akintunde@fuoye.edu.ng

Received September 26, 2018; Accepted October 25, 2018; Published October 31, 2018

Citation: Akintunde OA (2018) Distortion Operator of Uncertainty Claim Pricing Using Weibull Distortion Operator J Appl Computat Math 7: 425 doi: 10.4172/21689679.1000425

Copyright: $\odot 2018$ Akintunde OA. This is an open-access article distributed unde the terms of the Creative Commons Attribution License, which permits unrestricted use, distribution, and reproduction in any medium, provided the original author and source are credited. 


$$
g(x)=\left\{\begin{array}{l}
1, \text { if } u<a \\
0, \text { if } u \geq a
\end{array}\right.
$$

As shown in Figure 1 above, the distortion function is a step function with discontinuity at $1-\alpha$ and a big jump at $u=\alpha$. This discontinuity shows that $\mathrm{VaR}$ is not a coherent risk measure. The value $1-a$ the ruin or the expected shortfall. The conditional value at risk $((\mathrm{VaR})$ correspond to the distortion:

$$
g(x)\left\{\begin{array}{c}
1, \text { if } u<a \\
\frac{u-\alpha}{1-\alpha}, \text { if } u \geq a
\end{array}\right.
$$

The conditional value at risk $((\mathrm{VaR})$ as depicted is continuous but not differentiable at $\mathrm{u}=\alpha$ because of its zero value at this point. The distortion function $g$ maps all percentiles below a to a simple point 0 . This means that all the information in that part of the distribution is lost. Any smooty differentiable distortion $g$ will give a coherent risk measure that is different from $\mathrm{cVaR}$. A class of distortion based on the normal distribution in other to know the price for future returns of most risk assets was proposed as follows:

$$
\operatorname{Prg}(x)]=\int g S_{x}(x) d x=\phi\left[\phi^{-1}(u)+\alpha\right]
$$

Where in Figure $2 \varphi$ is the standard normal cumulative function (this is stated by Wang, $S, S_{0}$ ) the pricing kernel associated with this distribution is:

$$
\begin{aligned}
& H[x=h(z): \alpha]=\int_{-\infty}^{0} g_{\alpha} S_{\alpha} d x \\
& \text { And } \mathrm{E}[h(z+\alpha)]=S_{0} \mathrm{e}^{\alpha+\mathrm{a}}
\end{aligned}
$$

Where $\mathrm{h}$ is a continuous $\mathrm{lz}$ )sitive and increasing function. Clearly, for a normal random variable ,

$$
\begin{aligned}
& H[x=h(z): \alpha]=E[h(z+\alpha)] \\
& H[x=h(z):-\alpha] \Rightarrow H[S T-\alpha]=S_{0} e^{Z T+\alpha T}
\end{aligned}
$$

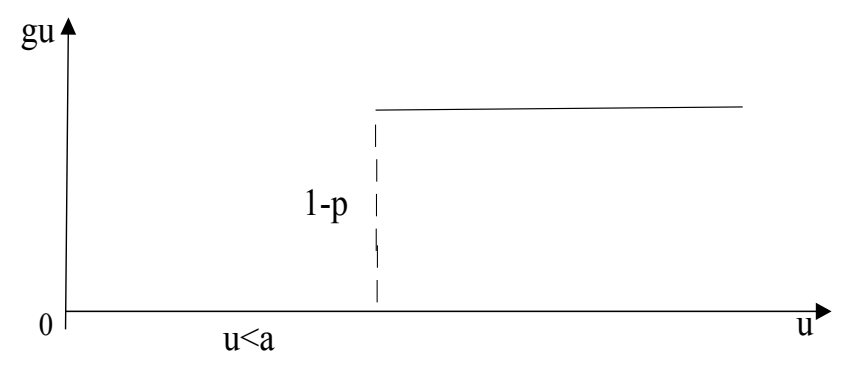

Figure 1: Distortion as a step function.

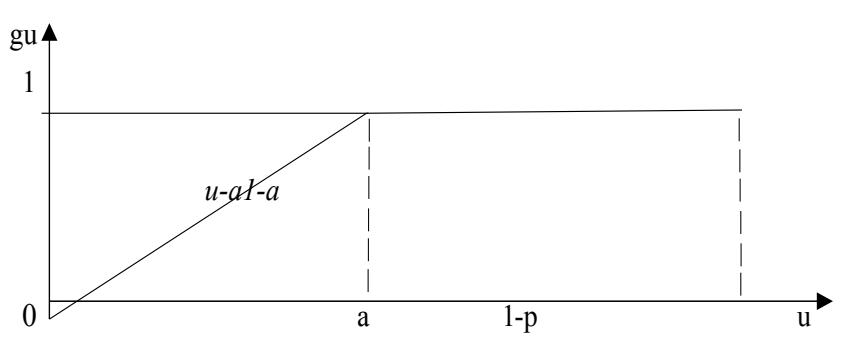

Figure 2: The conditional value at risk as distortion function.
Black-Scholes modelled the risk position S_1 of an asset at time by a geometric Brownian motion as:

$$
S_{1}=S_{0} e^{\left(N-\frac{\theta^{2}}{2}\right) t} w_{1} ; t \geq 0
$$

Where $\mathrm{w}$ is the Brownian motion.

For the pay-off of a European call option (with maturity $T$ and strike price $K$ ), we have:

$S_{T}=C\left(S_{T}, K\right)=\left(S_{T}-K\right)$

Where $S_{T}$ is a $\log$-normal random variable.

Applying kernel to this play-off with

$$
\alpha=\frac{u-r a \sqrt{T}}{\sigma}
$$

It can be shown that

$$
e^{-i T} H\left[c\left(x_{T}, K\right)\right]=S_{0} \phi\left[\ln \left(\frac{X_{0}}{K}\right)\right]+\left(\frac{r+\alpha^{2}}{2}\right) T-e^{-i T} K \phi \frac{\ln \left(\frac{Z_{0}}{K}\right)+\left(\frac{r+\alpha^{2}}{2}\right) T}{\sigma \sqrt{T}}=\sigma \sqrt{T}
$$

The risk free rate $\gamma$ shows that the Black-Scholes formula can be recovered from the distortion operator.

\section{The Weibull Distribution}

A generalized version of the distortion that is based on a Weibull distribution is introduced.

Definition: the Weibull distribution function is given by:

$$
f(x)=f(x, k, \beta, \alpha)=\frac{k}{\beta} \frac{(x-\alpha)^{k-1}}{\beta^{k-1}} e^{\frac{-(x-\alpha)^{k}}{\beta}}
$$

This consist of the failure and reliability notes [3]. $x$ is the loss random variable and $\alpha$ and $\beta$ are the scale parameter and $k$ is the shape parameter. The shape parameter determines the rate of the return and the scale parameter $\beta>0$ is proportional to the mean time failure.

Firstly, we consider the empirical survival function $S_{x}$, then the Weibull distortion operator is used to compute:

$$
\operatorname{Pr}[g(x)]=\int_{0}^{\infty} g S_{x}(x) d x
$$

We define the Weibull distribution operator as

$$
\begin{aligned}
& g_{x, k, \beta, \alpha}(u)=\varphi^{w} \\
& g_{x, k, \beta, \alpha}(u)=\phi^{w}\left[\phi^{w-1}(u)+\lambda\right]
\end{aligned}
$$

The same calibration; as in Wang distortion is adopted; firstly if a random variable has a Weibull distribution, its probability density function (pdf) is:

$$
f(x)=f(x, k, \beta, \alpha)=\left\{\begin{array}{c}
\frac{k}{\beta}\left(\frac{x-\alpha}{\beta}\right)^{k-1} e^{\frac{-(x-\alpha)^{k}}{\beta}}, x \geq 0 \\
0, x<0
\end{array}\right.
$$

Where $k>0, \alpha>0$. When $\alpha=0$, it reduces to two-parameter Weibull distribution. The failure return of the risky asset at $S_{T}$ is assumed to follow a Weibull distribution instead of the normal distribution.

$$
S_{T}=\mathrm{g}\left(Z_{\mathrm{t}}\right)
$$

Is the price of the security at time $\mathrm{T}$.

For a function $g(u)=S_{0} e^{u}$ of a random variable $Z_{t}$ with distribution web $(x, k, \beta, \alpha)$ implies $f(x, k, \beta, \alpha)$. 
$\left.H\left(S_{T}, \lambda\right)=\phi^{w-1}(u)+\lambda\right]=g x, k, \beta, \alpha S_{x}(x) d x$

Where $\phi^{w} s$ the Weibull distribution.

\section{Proposition}

Let $\mathrm{z}$ be a random variable and let $x=g(z)$ be the transformation of the continuous function $f(x)$ and $\phi^{w}=f(x, k, \beta, \alpha)=$ web $(x, k, \beta, \alpha)$ implies $f(\mathrm{x})$. Given that:

$$
\begin{aligned}
& S(x)=1-f(x)=\operatorname{Pr}(x>t) \\
& \text { or } S(x)=1=w e b(x, k, \beta, \alpha) \\
& \Rightarrow 1=\phi^{w}(x, k, \beta, \alpha)
\end{aligned}
$$

Then,

$$
H(x, \alpha)=\int_{0}^{\infty} g_{\alpha} S_{x}(x) d x=E[g(z+\lambda \beta)]
$$

\section{Proof}

Define

$H(x, \alpha)=\int_{0}^{\infty} g_{\alpha} S_{x}(x) d x=\phi^{w}\left[\phi^{w-1}(u)+\lambda\right]$

Where $S(x)=\operatorname{Pr}(x>t)=\operatorname{Pr}(g(x)>t)$

$$
\begin{aligned}
& =\operatorname{Pr}\left[\frac{g(Z)}{g}>\frac{t}{g}\right]=\operatorname{Pr}(z>g(t)) \\
& =1-\phi^{w}\left(g^{-1}(t)=\phi^{w}\left[-g^{-1}(t)\right]=u\right.
\end{aligned}
$$

Applying in eqn. (21) to the distortion function, we obtain

$$
\begin{aligned}
& \mathrm{G}(x, k, \beta, \alpha)\left(S_{x}(t)=\phi^{w}\left[\phi^{w-1}(u)+\lambda\right] \cdot \phi^{\mathrm{w}}\left[\phi^{w-1}\left(\phi^{w}\left[-\mathrm{g}^{-1}(t)\right]+\lambda\right]\right.\right. \\
& =\phi^{w}\left[-g^{-1}(t)+\lambda\right]=1-\phi^{w}\left(g^{-1}(t)-\lambda\right)
\end{aligned}
$$

The second application of probability and normalizing gives:

$$
\begin{aligned}
& \operatorname{Pr}\left[Z>g^{-1}(t)-\lambda\right]=\operatorname{Pr}\left[\frac{Z-\alpha}{\hat{a}}>\frac{g^{-1}(t)-\lambda-\infty}{\beta}\right] \\
& =\operatorname{Pr}\left[X>\frac{g^{-1}(t)-\lambda-\infty}{\beta}\right] \\
& =\operatorname{Pr}\left[x \beta>g^{-1}(t)-\lambda-\alpha \beta\right] \\
& =\operatorname{Pr}\left[x \beta>g^{-1}(t)-\lambda-\alpha \beta\right]
\end{aligned}
$$

Note: if $x=\frac{z-\alpha}{\beta}$

$x \beta=z-\alpha$

$z=x \beta+\alpha$

Applying the above, we get:

$\operatorname{Pr}[\mathrm{z} \beta+\alpha+\alpha \beta]>g^{-1}(t)$

$\operatorname{Pr}[\mathrm{z}+\alpha \beta]>g^{-1}(t)$

Multiplying through by $g$, we have:

$\operatorname{Pr}\left[g(z+\alpha \beta)>g^{-1}(t) \cdot g\right]=\operatorname{Pr}[g(z+\alpha \beta)>t]$

$=E^{n}[g(z+\alpha \beta)]$

Therefore,

$$
E_{x}^{n}=E^{n}[g(x+\alpha \beta)]=\int g_{\alpha} S_{x}(x) d x=E\left[S_{0} e^{z+\alpha \beta}\right]
$$

Where $E^{n}$ denotes the expectation under the density measure n.

$$
\text { So } H\left(S_{\mathrm{T}}=\lambda\right)=E\left[\mathrm{~S}_{0} e^{z+\alpha \beta} T\right]
$$

This result shows that under a $g_{x, k, \beta, \alpha}$ distortion, a Weibull random is translated by a force $\lambda \beta$. This generalizes the equivalent result found in normal Inverse Gaussian Distribution (NIG).

This implies $G\left[S_{\mathrm{t}}-\lambda\right]$ to:

$$
G\left[S_{T}-\lambda\right]=S_{0} e^{\Lambda t}
$$

Under Weibull distribution with value $\lambda \phi^{w}\left[\phi^{w-1}(u)+\lambda\right]$, the price at ST evolves like a risk neutral asset because $\lambda$ is calibrated to verify the risk neutral condition.

Hence,

$$
\begin{aligned}
& H\left[S_{t}-\lambda\right]=S_{0} e^{\lambda T}=\int g_{x, k, \beta, \alpha} S_{x}(t) d t \\
& =\phi^{w}\left[\phi^{w-1}(u)+\lambda\right]=E\left[S_{0} e^{\lambda+i \beta}\right] \\
& =E[g(z+\lambda \beta)]
\end{aligned}
$$

As required. This implies;

$G\left[S_{T}-\lambda\right]=E\left[S_{0} e^{\lambda t-\lambda \beta T}\right]$

The positivity of $g$ in $E[z+i \beta]$ can be relaxed. More generally, the proposition can be extended to the extended to the case when the security price is function of symmetrically distributed random variable. Apply the price $E[g(Z+\lambda \beta]$, the capital required for the standard call payoff is:

$$
\begin{aligned}
& G[F(S T, K) ; \lambda]=E[g(2 T+\lambda \beta)] \\
& =\left[S_{0} e^{2 T+\lambda \beta}-K\right] \\
& =\int_{-\infty}^{\infty}\left(S_{0} e^{2}-k\right) w e b(x, k, \beta, \alpha,+\lambda \beta) d t
\end{aligned}
$$

Having known the density function $G\left[F\left(S_{T}\right)\right]$ of the, stock price at the expiring time $S_{T}$ under the risk neutral measure $\lambda \beta$ we can easily price call and put option by simply calculating the expected value [4].

Hence, for a call option with strike price $\mathrm{k}$ at time $S_{T}$ to expiration $S_{T}$, the value at time 0 is therefore given by expectation of the payoff under the martingale measure ;

$$
\begin{aligned}
G(K, T)= & E_{0}[\exp (-\gamma T) \max (S T-K), 0] \\
= & \exp (-\gamma T) \int_{0}^{\infty} g_{0}(S, T) \max (S-K) d s \\
= & e^{-\gamma T} \int_{k}^{0} g_{Q}(S, T)(S-K) d s \\
& =e^{-\gamma T} \int_{k}^{0} g_{Q}(S, T) s d s-K e^{-i T} \pi
\end{aligned}
$$

Where $\pi$ is the probability under $\mathrm{Q}$ of the future prize of the risk asset,

$$
G(K, T)=\int_{Q \alpha}^{\infty}\left[\left(S_{0} e^{Z}-K\right) \frac{k}{\beta} Z^{k-1} e^{-Z K}+\alpha \beta\right] d z
$$

Then,

$$
\begin{aligned}
G\left[F\left(S_{T}, K\right)-\lambda\right]=\int_{Q \alpha}^{\infty}\left[\left(S_{0} e^{Z}-K\right) k Z^{k-1} e^{-Z K}-\lambda\right] d z \\
=\int_{Q \alpha}^{\infty}\left[S_{0} e^{Z} k z^{k-1} e^{-z k}-\lambda\right] d z-k \int_{Q \alpha}^{\infty}\left[k z^{k-1} e^{-z k}-\lambda\right] d z
\end{aligned}
$$


If $\lambda=\frac{\eta-r \epsilon}{\beta} T$

$S_{T}$ evolves as a risk neutral asset.

This shows that the price evaluated with $\mathrm{t} d$ : pricing kernel associated to the Weibull distortion with parameter is giving by:

$$
\begin{aligned}
& G[F(S T, K)-\lambda]=S_{0} e^{z} \int_{Q_{\alpha}}^{\infty} w e b\left[x, k, \beta_{T},(\propto+\lambda) T\right] d z-k \int w e b\left[x, k, \beta_{T},(\propto+\lambda) T\right] d z \\
& =S_{0} e^{z}\left(1-Q_{\alpha}^{w}-\lambda\right)-\left(1-Q_{\alpha}^{w}-\lambda\right) \\
& =S_{0} e^{2}\left(Q_{\alpha}^{w}+\lambda\right)-k\left(Q_{\alpha}^{w}+\lambda\right) \\
& \text { And } \\
& e^{-\mathrm{It}} G[F(S T, K)-\lambda]=S_{0} \\
& \mathrm{e}^{-\mathrm{iT}} \mathrm{G}[\mathrm{F}(\mathrm{ST}, \mathrm{K})-\lambda]-\mathrm{S}_{0} w e b\left[\mathrm{Q}_{\alpha, x, k(\alpha+\lambda)}\right]-K e^{-i T} w e b[\mathrm{Q} \alpha: x, k, \beta T,(\alpha+\lambda) T]
\end{aligned}
$$

Which is the price at the time $\mathrm{t}=0$. That is the price at $S_{T}$.

\section{Theorem}

If $Z_{t}$ has the Weibull distribution and a power function $\mathrm{g}(z)=Z^{\beta^{\prime}}$ then the optimal strategy has the power law distribution given by:

$$
H_{\beta}(z)=\frac{z^{1-\beta}}{\gamma \beta}
$$

\section{Proof}

Here, $Z_{t}$ is the continuous returns of a security. Metaz [5] had shown that it is possible to find an optimal investment strategy in terms of the probability density function describing the prices returns of a security. This strategy optimizes some appropriate measure of risk. Hence given our assumption of Weibull distribution of asset returns, we define the strategy that optimizes the variance of the distribution as:

$$
H_{p}(z)=\int_{0}^{\infty} g(z) d z
$$

Where $g(z)$ as in eqn.(15). Eqn. (34) reduces to:

$$
H_{p}(z)=\int_{0}^{\infty} \exp \left[-\gamma z^{\beta}\right] d z
$$

Set $x=\gamma z^{\beta}$, then $\frac{d x}{d z}=\gamma \beta z^{\beta-1}$ or $d z=\frac{1}{\gamma \beta} z^{1-\beta} d x$. Substitute this in eqn. (40), to get;

$$
\begin{aligned}
& H_{p}(z)=\frac{1}{\gamma \beta} z^{1-\beta} \int_{0}^{\infty} \exp (-x) d x \\
& =\frac{1}{\gamma \beta} z^{1-\beta}
\end{aligned}
$$

(As required).

\section{Conclusion}

Under Weibull distortion with value $\lambda=\frac{\eta-r c}{\beta}, \ln \phi^{w}\left[\phi^{w-1}(u)+\lambda\right]$, the price at future time $S_{T}$ evolves like a risk neutral risk neutral risk. $\lambda$ Is calibrated to verify the risk neutral condition.

The assumption that the prize of return at $S_{T}$ follows a Weibull distribution in place of normal distribution is based on its flexibility to follow the behaviour of other statistical distribution such as exponential and normal distribution. That is, other statistical distributions can be recovered from the Weibull distribution by change of the values of the parameters, in eqn. (38), (1- $\beta$ ) is the characteristic exponent of the generalized power law distribution. This shows that power law the generalized power law distribution. This shows that power law property relation characterizes the measure of risks modelled by the Weibull variables. The incurred risk measure of an investor faced with investment decision in eqn. (38).

\section{References}

1. Artzner P, Delbean F, Eber M, Heath D (1999) Coherent Measures of risk. Math Financ 9: 203-228

2. Wang SS (2000) A class of distortion operators for pricing financial and insurance risk. American Risk and Insurance Association 67: 15-36.

3. Osu BO (2009) Predicting changes in dynamic behaviour of asset price returns. J Math Sci, pp: 323-330.

4. Schoutens $W(2000)$ Levy processes in finance: pricing financial derivatives. John Wiley and sons Ltd, pp: 59-63.

5. Metaz A (2000) Financial modelling and option theory with the truncated levy process. International Journal of Theoretical and Applied Finance 3: 143-160.

6. Olkin I, Gloser JG, Derman C (2017) Probability models are applications Advance Information pp: 108-109. 